\title{
Quadratic controller for a chaotic micro-resonator
}

\author{
S. Towfighian \\ Mechanical Engineering \\ University of Waterloo \\ Waterloo, ON N2L 3G1, Canada \\ Email:stowfigh@engmail.uwaterloo.ca
}

\author{
G. R. Heppler \\ Systems Design Engineering \\ University of Waterloo \\ Waterloo, ON N2L 3G1, Canada \\ Email:heppler@uwaterloo.ca
}

\author{
E. M. Abdel-Rahman \\ Systems Design Engineering \\ University of Waterloo \\ Waterloo, ON N2L 3G1, Canada \\ Email:eihab@uwaterloo.ca
}

\begin{abstract}
A model for a chaotic micro-oscillator is developed by adding a new control system to an electrostatic actuator consisting of two electrodes: the substrate and a cantilever beam on top. The electrostatic actuator itself has a single stable equilibrium over a range of voltage, whereas adding the controller, which has a quadratic function, creates a voltage range for bistability. The bi-stability represents a two well potential field that is used here to create chaos. The model of the beam actuator is a mathematical model of a continuous system with identified parameters from the experiments. The equations of motion are then descretized using the first mode shape of the beam and solved numerically to find the steady state oscillation. The range of the $\mathrm{AC}$ frequency and voltage excitation for sustained chaos creation is investigated through bifurcation diagrams which reveal two well chaos, periodic orbits and multiple periods. It is discovered that the proposed new controller has some important advantages over a previously introduced controller for creation of chaos. First, it has a simpler function making its implementation easier. Second, it has a larger bi-stability voltage range starting from zero that doubles the operating range for chaotic oscillation and decreases the actuation voltage by eleven times. At last but not least, the controller offers chaos in a three times wider $\mathrm{AC}$ voltage range than that of the previous controller.
\end{abstract}

\section{INTRODUCTION}

Chaotic vibrations in Macro-scale were used to detect damages in the aeroelastic structures [1], [2] , and mass variation due to corrosion [3]. This type of nonlinear vibration is found to be very sensitive to small variations in some system parameters. In micro-scale; however, there are a number of studies reporting chaos experimentally [4], [5], in simulations [6], [7], [8], or both [9], [10], [11], [12]. The application of chaos in detection of system parameter changes in Microelectro-mechanical systems (MEMS) is a new open area of research that should be developed through a thorough study of chaotic vibrations in such devices. A number of these studies is performed on Atomic Force Microscopes (AFM) [10], [11], [4], a device for surface profile measurement operating based on an interaction of a micro-cantilever beam with a sample. These investigations were performed to identify and avoid chaotic regions in the parameter space to improve imaging quality and measurement accuracy. Chaos was observed in tapping mode AFM where the cantilever is driven around its natural frequency near the sample surface and its vibrations reveals the surface information [10], [11]. Raman et al. [4] also reported chaotic oscillation in AFM during the transition from noncontact to tapping regimes.
Chaos in electrostatic MEMS is also investigated in the last decade [6], [12], [5], [7], [8]. Developing an autonomous impact oscillator, Bienstman et al. [6] investigated the chaos creation in a capacitor by applying voltage larger than the pullin voltage. They reported chaos in the simulations and period doubling in the experiments. Wang et al. [12] simulated chaos in an electrostatic comb drive actuator using Duffing oscillator model. They observed chaos in a bi-stable state of the system. Adding feedback control system was also examined by Liu et al. [7] to create bi-stability leading to chaos. In an extension to his work, we [8] analyzed the system with an improved model, conducted a thorough study on the dynamics of the system, and presented the bifurcation diagrams to adjust the excitation voltage and frequency for producing sustained chaos. In this study, we present a new controller with enhanced performance for producing chaos. The system model with identified parameters from the experiment is presented followed by detailed static and dynamic analysis of the system.

\section{System MODELING}

The electrostatic actuator system is composed of one substrate electrode and a top electrode that is a cantilever beam. Voltage applied between the two electrodes is the driving force (Figure1). Using Euler-Bernoulli beam model, the open loop equation of motion in non-dimensional form becomes

$$
\frac{\partial^{2} w}{\partial t^{2}}(1-w)^{2}+\frac{\partial^{4} w}{\partial x^{4}}(1-w)^{2}+\mu \frac{\partial w}{\partial t}(1-w)^{2}=\alpha V_{D C}^{2}
$$

where the following non-dimensional parameters are used

$$
x=\frac{\hat{x}}{L}, \quad w=\frac{\hat{w}}{d}, \quad t=\frac{\hat{t}}{T}
$$

and $\hat{w}$ is the deflection of the beam in the $\hat{z}$ direction, $\hat{x}$ is the coordinate along the beam length, and $\hat{t}$ is time. The two introduced parameters of $\mu$ and $\alpha$ are given as

$$
\mu=\frac{c L^{4}}{E I T}, \alpha=\frac{\varepsilon_{0} b L^{4}}{2 E I d^{3}} .
$$

On the other hand, we have $\mu=2 \zeta \omega_{n}$, where $\zeta$ is the damping ratio found experimentally and $\omega_{n}=3.5158$ is the first canonical natural frequency of a cantilever beam. All other parameters are given in Table I.

The open loop system has been simulated to obtain the static response and the pull-in point where the system loses stability and hits the bottom electrode. The result is shown in Figure 

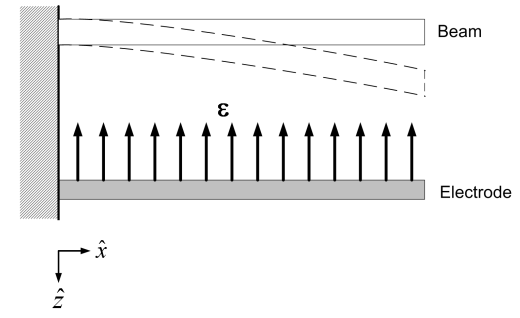

Fig. 1. Schematic of microbeam oscillator.

TABLE I

ACTUATOR PARAMETERS

\begin{tabular}{|l|c|c|}
\hline Parameter & Symbol & Value \\
\hline \hline Density & $\rho$ & $2331 \frac{\mathrm{kg}}{\mathrm{m}^{3}}$ \\
\hline Beam Length & $L$ & $150.6 \mu \mathrm{m}$ \\
\hline Beam Width & $b$ & $9.9 \mu \mathrm{m}$ \\
\hline Beam height & $h$ & $1.9 \mu \mathrm{m}$ \\
\hline Initial gap & $d$ & $2 \mu \mathrm{m}$ \\
\hline Damping coefficient & $\mu$ & 0.497 \\
\hline Permittivity of free space & $\varepsilon_{0}$ & $8.85 \mathrm{E}-12 \frac{\mathrm{F}}{\mathrm{m}}$ \\
\hline Modulus of Elasticity & $E$ & $150 \mathrm{GPa}$ \\
\hline
\end{tabular}

2 that predicts pull-in happens at 25.3 Volts when the top electrode reaches 46 percent of the initial gap.

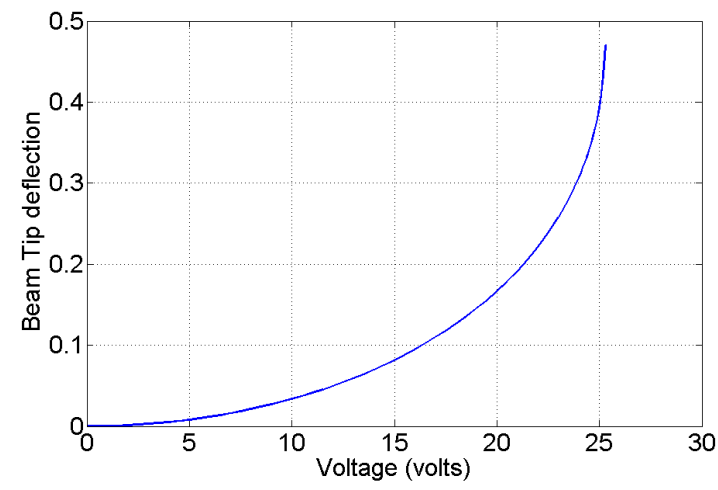

Fig. 2. Open loop static response.

To create a chaotic actuator, a controller is added to the micro-oscillator to make it a bi-stable system (Figure 3). By adding the controller, the equation of motion changes to

$$
\ddot{w}(1-w)^{2}+w^{(4)}(1-w)^{2}+\mu \dot{w}(1-w)^{2}=\alpha\left(V_{\text {ref }}-\hat{V}_{c}\right)^{2}
$$

where $\ddot{w}$ and $\dot{w}$ are second and first derivatives with respect to time and $w^{(4)}$ is the fourth derivative with respect to the coordinate along the length. $\hat{V}_{c}$ is the output voltage of the controller, and $V_{\text {ref }}$ is the input voltage:

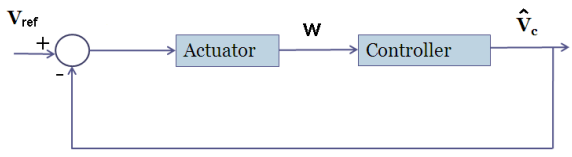

Fig. 3. Controller system .

$$
V_{\text {ref }}=V_{D C}+V_{A C} \cos (\omega t)
$$

where $V_{D C}$ and $V_{A C}$ are applied $\mathrm{DC}$ and $\mathrm{AC}$ voltages respectively with non-dimensional circular excitation frequency of $\omega$. The controller voltage rate is composed of a quadratic function of the normalized beam tip deflection $w(1)$ and an integrator with a constant gain of $K_{I}=270$ :

$$
\dot{\hat{V}}_{c}=-K_{I}\left(\hat{V}_{c}-\left(a_{0}+a_{1} w(1)+a_{2} w^{2}(1)\right)\right)
$$

where $a_{0}, a_{1}, a_{2}$ are the coefficient of the quadratic function. To obtain the static and dynamic responses, the partial differential Eq. (4) is converted to an ordinary differential equation using separation of variables and Galerkin's method using one mode shape of the beam as described in Towfighian et al.[13]. The beam deflection function is thus

$$
w(x, t)=\Phi_{1}(x) q_{1}(t)
$$

where $\Phi_{1}(x)$ is the 1st cantilever beam mode shape normalized with respect to the beam tip modal deflection so that $\Phi_{1}(1)=$ 1 , and $q_{1}$ is the normalized beam tip deflection. A set of second order ordinary differential equations is then obtained:

$$
\left\{\begin{array}{l}
\left(\ddot{q}_{1}+\mu \dot{q}_{1}+\omega_{1}^{2} q_{1}\right)\left(1+c_{1} q_{1}+c_{2} q_{1}^{2}\right)=c_{3}\left(V_{r e f}-\hat{V}_{c}\right)^{2} \\
\dot{\hat{V}}_{c}=-K_{I}\left(\hat{V}_{c}-\left(a_{0}+a_{1} q_{1}+a_{2} q_{1}^{2}\right)\right)
\end{array}\right.
$$

where $\omega_{1}$ is the first natural frequency of the beam, and $c_{1}$, $c_{2}$, and $c_{3}$ are found after applying Galerkin's method.

\section{A. Experimental Parameter identification}

The actuator was designed using the PolyMUMPs, 3 dimension profiler scanning of which is shown in Figure 4. The beam dimensions including width, thickness and electrodes gap are measured using the optical profiler. The experimental beam natural frequency is found to be $100.246 \mathrm{kHz}$ using a Miscroscope scanning vibrometer (Polytec MSV-400). To account for the flexibility in the support, the beam length is adjusted to $156 \mu \mathrm{m}$ to match the experimental natural frequency and the modulus of elasticity of $150 \mathrm{GPa}$. The damping ratio has also been found experimentally to be $\zeta=0.0707$.



Fig. 4. Optical profilometer 3D scan of the actuator (Vision Software).

\section{STATIC ANALysis}

The static response of the system after adding the controller is obtained by setting the time derivative of Eq. (8) to zero and solving numerically. Figure 5 shows the beam tip deflection 
versus applied voltage. For examining the stability of equilibrium points at different voltages, the system of equations are linearized around the equilibrium points and the eigenvalues are obtained at those points. Eigenvalues found at $V_{D C}=10 \mathrm{~V}$ of Figure 5 is listed in Table II. As one can see, the first and third equilibrium points are stable points while the second and fourth equilibrium points are saddles. The bi-stability voltage range starts at zero and ends at $11.7 \mathrm{~V}$, above which there only exists an upper saddle and a lower stable equilibrium point. The bi-stability DC voltage range for the new controller is 2.4 times the range of previous controller [8] which expands the region for chaos production. As the voltage is increased from zero, the distance of the upper saddle from the upper stable equilibrium increases that reduces the risk of touching the unstable manifold of the saddle and pull in making the chaotic oscillation safer to operate between the two potential wells. Thus, DC voltage of $10 \mathrm{~V}$ is chosen for the operation of the chaotic actuator from this point forward. Table II also reveals the non-dimensional natural frequencies of the first and third equilibrium points as 13.89 and 7.88 corresponding to $396 \mathrm{KHz}$ and $224.7 \mathrm{KHZ}$ respectively.

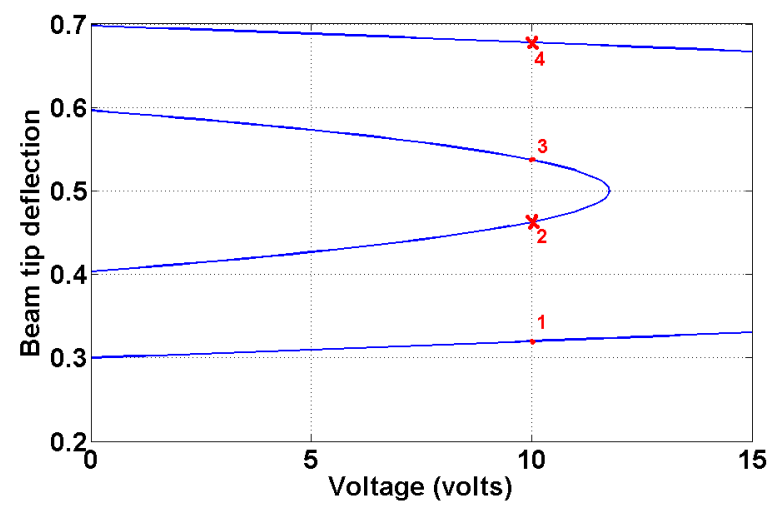

Fig. 5. Non-dimensional deflection of the beam tip versus voltage.

TABLE II

EIGENVALUES FOR THE EQUILIBRIUM POINTS AT $V_{\text {ref }}=10 \mathrm{~V}$ IN FIGURE 5.

\begin{tabular}{|c|c|c|c|}
\hline$\#$ & $q_{1}$ & Eigenvalue 1 & Eigenvalue 2 \\
\hline \hline 1 & 0.3198 & $-0.2485-13.8868 \mathrm{i}$ & $-0.2485+13.8868 \mathrm{i}$ \\
\hline 2 & 0.4625 & 7.0365 & -7.5335 \\
\hline 3 & 0.5372 & $-0.2485-7.8759 \mathrm{i}$ & $-0.2485+7.8759 \mathrm{i}$ \\
\hline 4 & 0.6778 & 20.0625 & -20.5595 \\
\hline
\end{tabular}

\section{Dynamic Analysis}

The dynamic response of the system to the reference input voltage $V_{\text {ref }}$ including DC and AC components (Eq. (5)) is obtained by numerically integrating Eqs. (8) for the duration of 5000 times the excitation period. For obtaining the bifurcation diagrams, the response is examined once the transients disappear and during the last 128 periods when either the frequency or the amplitude of AC voltage is swept. At each value of the control parameter, a poincare section is constructed by starting at the maximum displacement and sampling the orbit once every period.

\section{A. Force sweep}

Figure 6 demonstrates the bifurcation diagram obtained from sweeping the AC amplitude at fixed DC voltage $V_{D C}=$ $10 \mathrm{~V}$, and the excitation frequency of $\omega=10.1$. Increasing the amplitude from $2 \mathrm{~V}$, first we observe periodic orbits between the two potential wells up to $2.6 \mathrm{~V}$. Beyond that, periodic orbit along with weakly chaotic orbits coexists in the lower well. In the range of 3.95-4.3 V, the solution is exclusively chaotic oscillations between the two wells. In comparison of the new quadratic controller to the previously developed controller [8], the proposed controller offers a three times wider AC voltage range for producing two well chaotic oscillation which makes it easier to implement. One can also observe a large periodic orbit between the two wells around $4.3 \mathrm{~V}$ that becomes weakly chaotic coexisting with the two well chaotic attractors. Beyond $4.69 \mathrm{~V}$, the energy seems to become high enough that can not be consumed between the two wells, the orbit jumps over the hump or the upper saddle and dynamic pull-in occurs. A phase portrait of the chaotic attractor at $V_{A C}=4 \mathrm{~V}$ beside the controller output and the actuator input voltage changes are shown in Figure 7. The random oscillation between the two wells and individual oscillations in each well are observed in the phase portrait. In the bottom left graph, the range of controller output voltage is between -55 to $32 \mathrm{~V}$ and the actuator input voltage changes in the range of -24 to 63 V. Comparing the previous controller[8], the new controller has significantly reduced the reference voltage, $10 \pm 4 \mathrm{~V}$ vs $110 \pm 4 \mathrm{~V}$ and the maximum controller voltage, $55 \mathrm{~V}$ vs. 80 $\mathrm{V}$, which makes the fabrication much simpler.

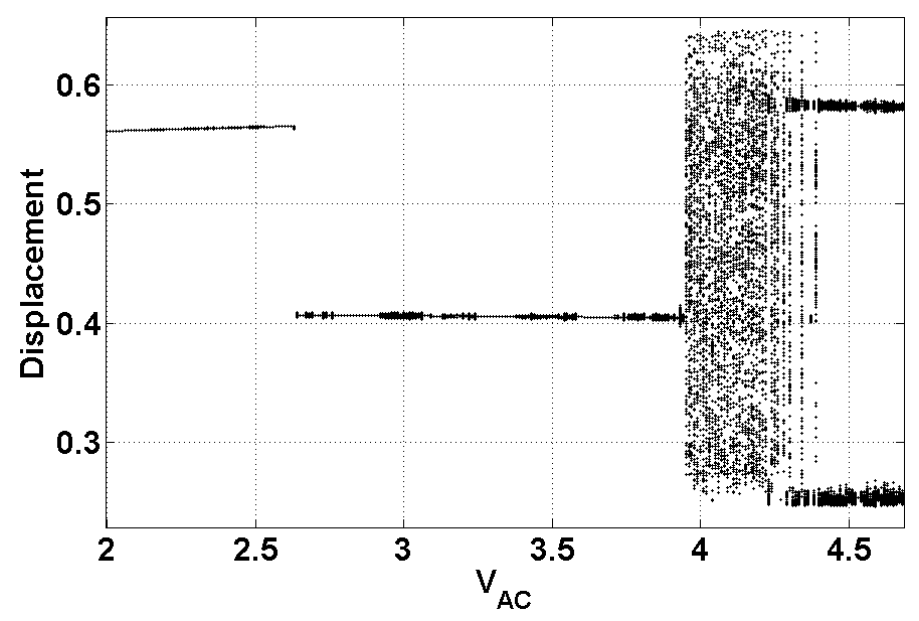

Fig. 6. Bifurcation diagram constructed from a force sweep $V_{A C}$ at fixed frequency of $\omega=10.1$.

\section{B. Frequency sweep}

Sweeping the frequency of excitation around $\omega=10$, while keeping the AC constant at $V_{A C}=4 V$, we obtain Figure 8. The figure illustrates a two well chaotic attractor well 

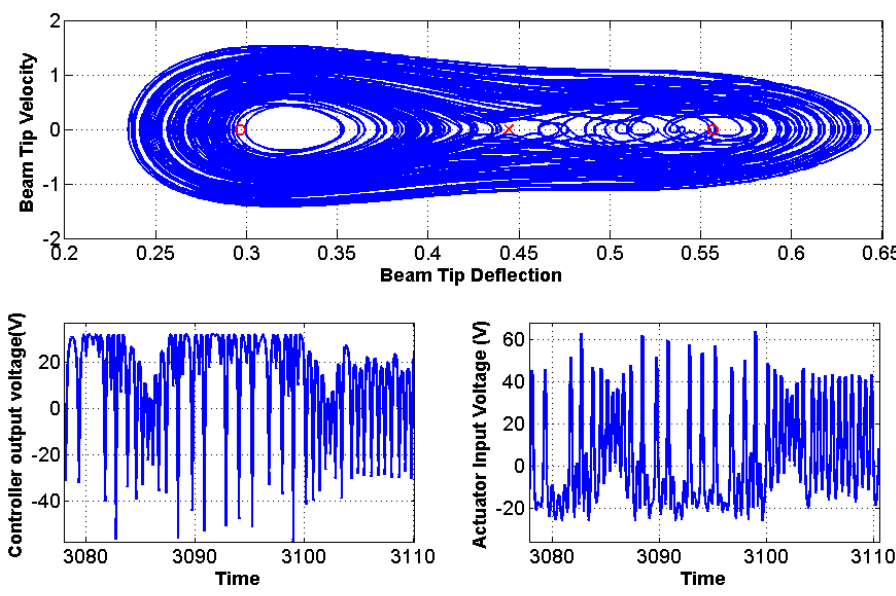

Fig. 7. Top: Phase portrait of chaotic oscillation, Bottom left: controller output voltage $(\mathrm{V})$, Bottom right: actuator input voltage $(\mathrm{V})$ once the amplitude of excitation $V_{A C}=4$ and the non-dimensional frequency of excitation $\omega=10.1$.

developed in a wide range of excitation frequency between 9.71 and 10.18 followed by lower-well periodic orbits. There also exists a narrow chaotic region from 11.13 to 11.27 , above which the bifurcation changes to Period 4 between the two wells. The frequency range for producing chaotic oscillation using the new proposed controller is twenty percent larger than the range obtained in [8].

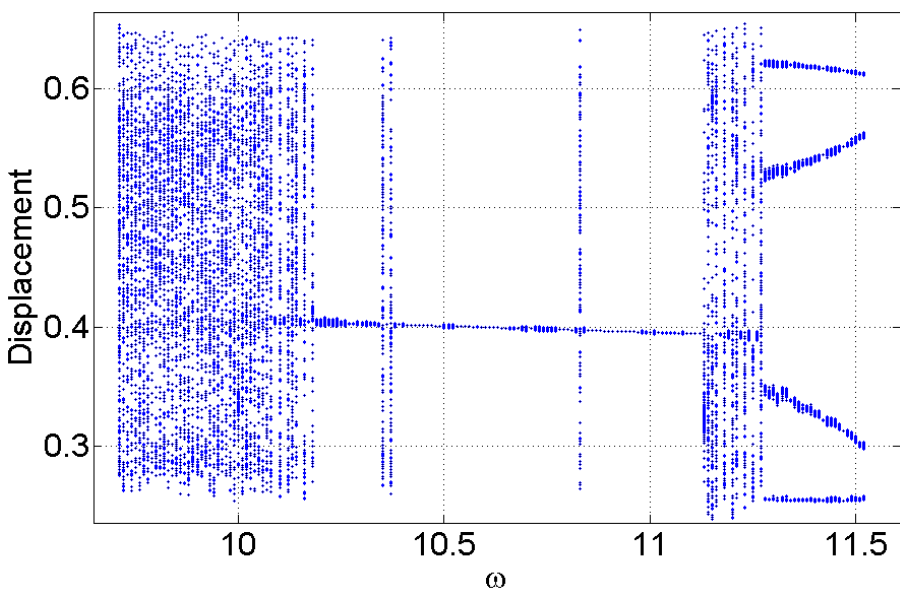

Fig. 8. Bifurcation diagram sweeping the frequency of excitation at fixed $V_{A C}$ of $4 \mathrm{~V}$.

\section{CONCLUSiON}

A new quadratic controller is added to an electrostatic actuator for creation of sustained chaotic vibraions. The system is composed of an electrode underneath and a microbeam on top that is actuated by applying a voltage. The continuous mathematical model of the beam is descretisized using the first mode shape of the cantilever beam. The added controller converts the beam actuator to a bi-stable system for a range of DC voltage, which is used to produce chaos. The dynamic behaviour is investigated and results of steady state vibrations are presented in bifurcation diagrams. The diagrams suggest that the new controller in comparison to the previously developed controller [8], has some advantages that includes three times larger operating range for $\mathrm{AC}$ voltage amplitude and twenty percent larger range for $\mathrm{AC}$ frequency. The wide operating ranges are desired for implentation of the device. In addition, the chaotic vibration can be created at eleven times less DC actuation voltage. Finally, the controller requires less voltage than the previous one making its design simpler. The chaotic actuator developed will be further investigated to be used for detecting or sensing in MEMS.

\section{ACKNOWLEDGMENT}

This research was financially supported by Natural Sciences and Engineering Research Council of Canada (NSERC) and Canadian Foundation for Innovation(CFI). The authors also would like to thank Mohammad Basha, Kamran Shavezipur, and Sara Sharifian for their help in this project.

\section{REFERENCES}

[1] B. I. Epureanu, L. S. Tang, and M. P. Paidoussis. Exploiting chaotic dynamics for detecting parametric variations in aeroelastic systems. AIAA Journal, 42(4):728-735, 2004.

[2] B. I. Epureanu, S. H. Yin, and M. M. Derriso. High-sensitivity damage detection based on enhanced nonlinear dynamics. Smart Materials and Structures, 14(2):321-327, April 2005.

[3] S.-H. Yin and B.I. Epureanu. Experimental enhanced nonlinear dynamics and identification of attractor morphing modes for damage detection. Journal of Sound and Acoustics, 129(6):763-770, Dec. 2007.

[4] A. Raman and S. Hu. Chaos in dynamic atomic force microscopy. In 2006 International Symposium on Nonlinear Theory and its Applications, pages 911-14, Bologna, Italy, 2006.

[5] B.E. DeMartini, H.E. Butterfield, J. Moehlis, and K.L. Turner. Chaos for a microelectromechanical oscillator governed by the nonlinear mathieu equation. Journal of Microelectromechanical Systems, 16(6):1314-1323, Dec. 2007.

[6] J. Bienstman, J. Vandewalle, and R. Puers. Autonomous impact resonator: A new operating principle for a silicon resonant strain gauge. Sensors and Actuators, A (Physical), 66(1-3):40-49, 1998.

[7] S. Liu, A. Davidson, and Q. Lin. Simulation studies on nonlinear dynamics and chaos in a MEMS cantilever control system. Journal of Micromechanics and Microengineering, 14(7):1064-1073, 2004.

[8] S. Towfighian, G. R. Heppler, and E. M. Abdel-Rahman. Analysis of a chaotic electrostatic micro-oscillator. Submitted to Jouranl of Computational and Nonlinear Dynamics, Apr. 2009.

[9] M. Basso, L. Giarré, M. Dahleh, and I. Mezic. Numerical analysis of complex dynamics in atomic force microscopes. In Proceedings of IEEE International Conference on control Applications, pages 10261030, Trieste, Italy, Sep. 1998.

[10] M. Ashhab, M.V. Salapaka, M. Dahleh, and I. Mezic. Melnikov-based dynamical analysis of microcantilevers in scanning probe microscopy. Nonlinear Dynamics, 20(3):197-220, Nov. 1999.

[11] M. Ashhab, M.V. Salapaka, M. Dahleh, and I. Mezic. Dynamical analysis and control of microcantilevers. Automatica, 35(10):1663-1670, Nov. 1999.

[12] Y. C. Wang, S. G. Adams, J. S. Thorp, N. C. MacDonald, P. Hartwell, and F. Bertsch. Chaos in MEMS, parameter estimation and its potential application. IEEE Transactions on Circuits and Systems I: Fundamental Theory and Applications, 45(10):1013-1020, 1998.

[13] S. Towfighian, E. M. Abdel-Rahman, and G. R. Heppler. Static and dynamic analysis of a bistable micro-actuator. In Proceedings of ASME International Mechanical Engineering Congress and Exposition, pages 1-11, Boston, MA, USA, 2008. 